\title{
Low Loss Nanophotonic Waveguides and Ring Resonators in Silicon-on-Insulator
}

\author{
Roel Baets ${ }^{1}$, Wim Bogaerts ${ }^{1}$, Dirk Taillaert ${ }^{1}$, Pieter Dumon ${ }^{1}$, \\ Peter Bienstman $^{1}$, Dries Van Thourhout ${ }^{1}$, Joris Van Campenhout ${ }^{1}$, \\ Vincent Wiaux ${ }^{2}$, Johan Wouters ${ }^{2}$, Stephan Beckx ${ }^{2}$ \\ ${ }^{I}$ Ghent University - IMEC, Dept. of Information Technology, Sint-Pietersnieuwstraat 419000 Gent, \\ BELGIUM,Wim.Bogaerts@intec.ugent.be \\ ${ }^{2}$ IMEC vzw, Silicon Process Technology Division, Kapeldreef 75, 3001Leuven, BELGIUM
}

\begin{abstract}
High index contrast, wavelength-scale structures are the key to ultra-compact integration of photonic integrated circuits. We discuss the general properties of these nanophotonic components and the rationale for using them. We studied the fabrication of these structures in the Silicon-on-insulator material system using CMOS processing methods and find this technology to be suitable for commercial implementation of nanophotonic IC's. We review photonic wires as one of the paths towards achieving denser integration and discuss the measurement results of waveguides and ring resonators. To tackle the coupling to fiber and the polarization dependency problem, we studied and fabricated vertical fiber couplers.
\end{abstract}

\section{INTRODUCTION}

Integration of photonic functions in a chip can bring the same massive advantages to photonics as what integration has done in the field of micro-electronics: cost reduction through high yield wafer scale processes, high performance, compact implementation etc. Furthermore in photonic integrated circuits (PICs) the alignment of subcomponents on a chip is automatically achieved through the lithographic processes, thereby reducing the need for active alignment methods which are notorious for dominating the cost of discrete optoelectronic components.

The promise of photonic integration - possibly large scale integration - is jeopardized however by the typical size of today's photonic components. Many components have a length scale of several 100 microns to several millimeters and in some cases even several centimeters. This is not only true for active components but also for passive components such as filters or (de)multiplexers and even for simple interconnecting waveguides such as bends, couplers and splitters.

One of the reasons for this scale problem is that most of today's integrated waveguide systems make use of rather weak confinement: the guided light beam is only one of many electromagnetic modes sustained by the system. This means that the entire system needs to be carefully designed such that all generated light stays within the guided mode(s) and does not couple to the sea of radiation modes. This leads to large components. 
In order to drastically reduce the size of photonic components we have to work with waveguides with only few resonant electromagnetic modes. This is possible by making use of waveguides with a very strong refractive index contrast - of the order of 2 to 1 - between core and cladding and a transverse cross-section of the order of the wavelength or less. The confinement in such waveguides can be either due to total internal reflection as in conventional waveguides or to Bragg diffraction as in photonic crystal waveguides.

This move to ultra-compact waveguide circuits comes at a prize however: the geometrical features become very small and have to be very accurate. The feature sizes encountered in such waveguides range typically from 50 to $500 \mathrm{~nm}$ and their accuracy needs to be in the range of 1 to $10 \mathrm{~nm}$. Therefore these waveguides are being called nanophotonic waveguides. It is clear that this calls for the use of very advanced fabrication techniques.

In this chapter we will review our efforts to make high quality nanophotonic circuits in Silicon-on-Insulator by means of mainstream technologies used for the manufacturing of advanced CMOS circuits.

In the next paragraph some of the basic mechanisms underlying high index contrast waveguides are covered. In particular the rationale for high index contrast in the inplane direction and the out-of-plane direction will be discussed. This is followed by a discussion of the technology - deep UV lithography and dry etching - for nanophotonic waveguide structures. The losses obtained in single mode waveguides with widths in the range of 400-500 $\mathrm{nm}$ will then be described. In the next paragraph we show that this technology allows for the fabrication of low loss micro-ring resonators with a radius of typically 5 micrometer. Finally we describe a novel approach for coupling light between the nanophotonic chip and standard optical fibers. This approach also allows dealing with the polarization dependence of high index contrast waveguides.

\section{RATIONALE FOR HIGH INDEX CONTRAST WAVEGUIDES}

This paragraph starts by reviewing a set of simple considerations with respect to high index contrast waveguides. We will start with the simplest possible waveguide: the symmetric slab waveguide. Actually this slab waveguide can also represent the very simplest model for a Fabry-Pérot resonator and therefore our discussion serves two purposes.

An electromagnetic field in any of the three layers can be Fourier-decomposed into an infinite set of plane-wave-like components each characterized by a k-vector. In a kspace representation these k-vector components will, for a given frequency, form spheres the radius of which scales with the refractive index. Hence we need to consider two spheres in this case, one for the core and one for the cladding. A k-vector in the core can only exchange energy with a k-vector in the cladding if they have identical $\mathrm{k}_{\mathrm{x}}$-components. This means that the $\mathrm{k}$-vectors in the core belong to two classes: those that cannot exchange energy with the cladding k-vectors (strictly guided contributions confined by total internal reflection) and those that can exchange energy. The latter form a double cone as indicated by the dashed region in Fig. 1. The higher 
the index contrast between the core and the cladding is, the smaller the solid angle of these cones and therefore the smaller the fraction of k-vectors that can couple to radiating fields.

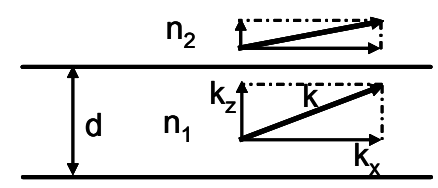

$\mathrm{n}_{2}$

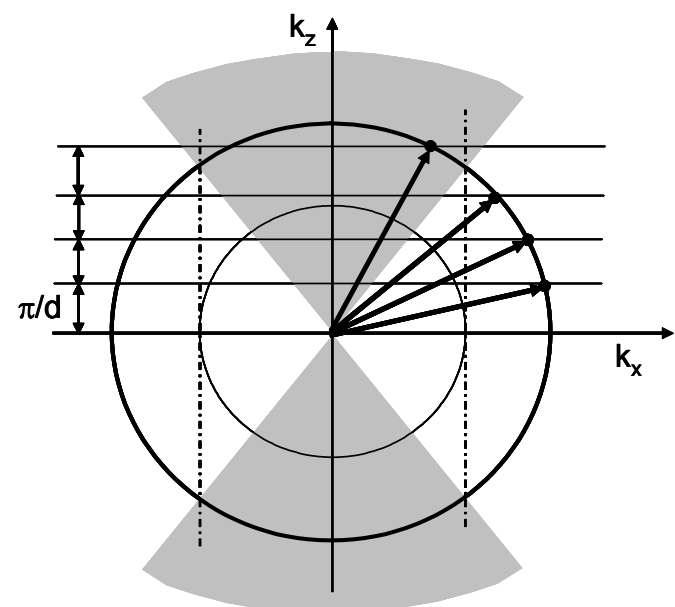

FIGURE 1. Slab waveguide / resonator and a k-space representation of the resonant guided and radiating modes

The strong reflections at the high index contrast interfaces will lead to strong resonances if the $\mathrm{k}_{\mathrm{z}}$-component in the core satisfies

$$
k_{z}=m \frac{\pi}{d}
$$

This is actually an approximate formula that does not take into account the phase shift associated with the reflection at the interface between core and cladding (due to either total reflection or due to complex refractive indices). Any generation or scattering of light will preferentially couple to these resonant modes. They are indicated in Fig. 1 by the bold arrows (each of which actually represents a cone in this simple 1D geometry). As one can see both guided and radiating resonances may exist in the structure. The guided resonances are generally called guided modes. The radiating resonances are the Fabry-Pérot modes of the cavity.

In order to limit the number of such resonances the thickness $d$ clearly needs to be small. Furthermore it is often desirable to work with waveguides with only one guided mode. This leads to the following condition for the maximum thickness as a function of the index ratio:

$$
d=\frac{\lambda / 2 n_{1}}{\sqrt{1-\left(\frac{n_{2}}{n_{1}}\right)^{2}}}
$$

This leads to the conclusion that a high index contrast single-mode waveguide needs to have a thickness of the order of half the wavelength in the material or less. 
It is worth considering how a deviation in the thickness $d$ affects the spectral behavior of a cavity or waveguide. A cavity-normal Fabry-Pérot mode will undergo a shift of its resonance wavelength due to a thickness deviation as given by:

$$
\frac{\delta \lambda}{\lambda}=-\frac{\delta k_{z}}{k_{z}}=\frac{\delta d}{d}
$$

If the thickness $d$ is of the order of the wavelength - as needed for a system with few modes - this means that the tolerable thickness inaccuracy is of the same order as the tolerable resonance wavelength inaccuracy.

In wavelength dependent waveguide circuits (filters, demultiplexers...) the spectral transmission will also shift as a result of a deviation of the waveguide thickness. In most types of circuits the relative wavelength shift will equal the relative change in effective index. This leads to the equation:

$$
\frac{\delta \lambda}{\lambda}=-\frac{\delta k_{x}}{k_{x}}=\frac{k_{z}^{2}}{k_{x}^{2}} \frac{\delta k_{z}}{k_{z}}=-\frac{k_{z}^{2}}{k_{x}^{2}} \frac{\delta d}{d}
$$

Again the conclusion is that in high index contrast wavelength-scale waveguides the tolerable thickness deviation is of the same order as the tolerable wavelength error. If the wavelength needs to have an accuracy of, say, $1 \mathrm{~nm}$, then the structural dimensions will also need to have accuracy of the order of $1 \mathrm{~nm}$. Moreover, the relative wavelength shift also scales with $\mathrm{k}_{\mathrm{z}} / \mathrm{k}_{\mathrm{x}}$, which is typically much larger in high contrast waveguides, meaning a much higher relative accuracy is needed in high contrast systems.

While this discussion has been limited to the very simple case of a one-dimensional structure, it is clear that the conclusions will - at least qualitatively - also hold for more complex three-dimensional waveguide or cavity structures with wavelength-scale dimensions.

So far the key argument in favor of wavelength scale high-index-contrast waveguides/cavities is that an electromagnetic field excited (or scattered) within the waveguide or cavity will mainly couple to a small set of electromagnetic modes. There are several other arguments in favor of structures with high refractive index contrast. High index contrast structures allow making high reflectivity mirrors with a broad spectral and angular range. By arranging such mirrors in a periodic arrangement one can build photonic band gap structures. A further and potentially very important property of wavelength-scale cavities and waveguides is that they allow for very high field strengths (or power densities) at modest power level. This means that the weak non-linearities present in many materials can be enhanced by many orders of magnitude and can therefore lead to practical non-linear devices.

Let us now look at the structure of real-world high index contrast waveguides for photonic IC's. Figure 2 shows the two generic types of such waveguides: in the first type the guided mode confinement is provided by total internal reflection (TIR) 
whereas in the second type the confinement is brought by a combination of total internal reflection and photonic band gap effects. In both cases a high in-plane index contrast is achieved by etching through the entire core of a high index material, typically a semiconductor. This allows for making very compact bends, couplers, resonators etc.
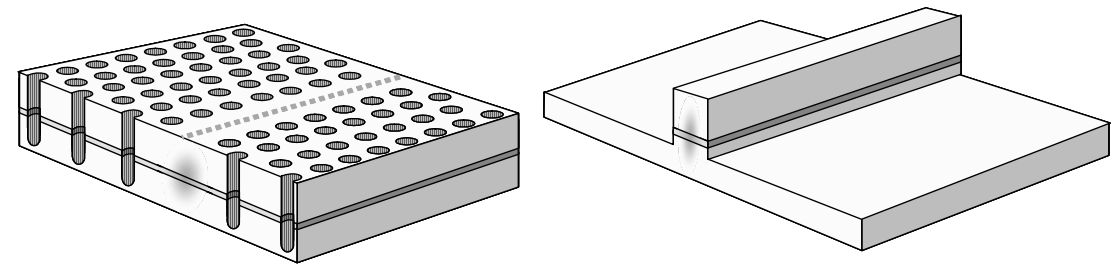

FIGURE 2. High index contrast photonic crystal defect waveguide (left) and TIR waveguide (right)

In these structures the out-of-plane index contrast can be chosen either low or high. It is a non-trivial design issue to analyze the relative merits of both options. While a low-index contrast approach is more compatible with conventional device structures based on III-V semiconductor heterostructures, in particular when it comes to making electrically driven active devices, the high-index contrast approach brings a number of important advantages, certainly for passive optical functions.

First of all, high out-of-plane index contrast implies that the core will be very thin and that the etch depth required to define the waveguides is rather small. Therefore the aspect ratio of the features to be etched will be of the order of 1 to 3 whereas in the case of low out-of-plane index contrast it increases to ratios of 5 to 10 . Secondly it has been shown that high out-of-plane index contrast structures are less sensitive to roughness of the etched sidewalls [1] than low contrast structures. Thirdly the verticality of these sidewalls is much less critical in high-index contrast structures than in low-index contrast structures. All these factors imply that the technology for high index contrast waveguides is much simpler than that for low-index contrast waveguides and that the losses due to roughness induced scattering in photonic crystal waveguides as well as out-of-plane radiation losses in photonic crystal and TIR waveguides are much lower, a fact which is clearly supported by state-of-the-art experimental results.

Other arguments in favor of high out-of-plane index contrast include the fact that as discussed before - the tighter vertical confinement will lead to higher field strength for a given optical power and also that certain functions, such as the compact vertical fiber coupler discussed in the last section, are only possible when using high index contrast structures.

A very suitable material system for passive nanophotonic circuits for use in telecommunication applications is the Silicon-on-Insulator (SOI) system. The waveguide core in this system consists of Silicon (with a refractive index of 3.48 at $1550 \mathrm{~nm}$ ) and the cladding is either silica (with a refractive index of 1.44) or air (or an asymmetric combination of both). Given their relevance for microelectronics applications SOI wafers of very high quality are industrially produced. Furthermore one can envision that the processing technologies used for the fabrication of submicron CMOS circuits are also usable for nanophotonic circuits. This would then lead to a direct roadmap for low cost manufacturing of photonic ICs. 
From the discussion so far one can conclude that there are two major arguments to study SOI-based nanophotonic ICs by means of CMOS technologies. The first argument is optical in nature: high index contrast (in both transverse dimensions) opens up the potential for very compact and possibly highly integrated circuits. The second argument is economical: using CMOS technology for nanophotonic circuits brings the potential of low cost manufacturing (whereby fabless operation becomes a real option for component companies) and, if needed, volume manufacturing.

In the next paragraphs we will describe the achievements in our research towards that goal.

\section{FABRICATION WITH HIGH-END CMOS PROCESSES}

For our high-contrast photonic structures, we use Silicon-on-Insulator. The top Silicon layer provides a good waveguide core, because its high refractive index $(n=3.48)$ contrasts very well with the underlying oxide $(n=1.44)$. The function of the oxide is here to optically separate the guiding top layer from the substrate, which has a similar large refractive index. Therefore, the oxide should be thicker than needed for CMOS applications [2]. For compatibility with the fabrication equipment, $200 \mathrm{~mm}$ wafers were used. We used UNIBOND wafers fabricated by SOITEC [3] with a Silicon layer thickness of $220 \mathrm{~nm}$ and a silica buffer of $1 \mu \mathrm{m}$.

The fabrication process with deep UV lithography is similar to that of conventional optical lithography or e-beam lithography. The process is illustrated in Fig. 3. First, a photoresist is coated on top of a $200 \mathrm{~mm}$ SOI wafer, and then pre-baked. On top of the resist, an anti-reflective coating is spun to eliminate standing waves in the photoresist. Then the wafer is sent to the stepper, which illuminates the photoresist with the pattern on the mask. As a $200 \mathrm{~mm}$ wafer can contain many structures, the die with the pattern is repeated across the wafer. This can be done with varying exposure conditions. After lithography, the resist goes through a post-exposure bake, and is then developed. For our experiments we used Shipley UV3 resist, opening up the exposed areas.

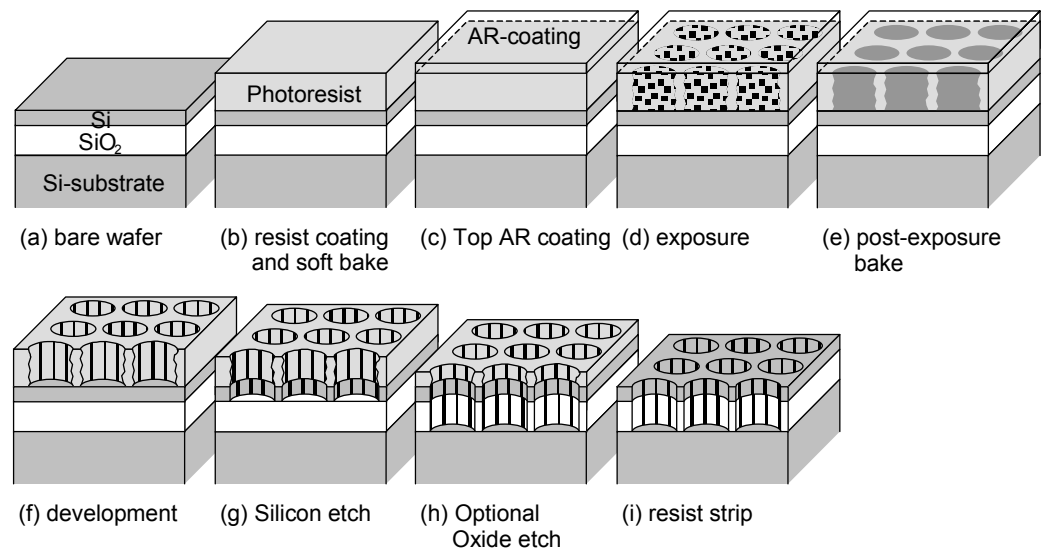

FIGURE 3. Fabrication of photonic nanostructures in SOI with deep UV lithography and dry etching. 
The developed photoresist is then used directly as a mask for etching. We can either etch just the top Silicon layer, or etch through both the Silicon and the oxide layer. Also, a number of post-processing steps are possible, including thermal oxidation or oxide deposition.

\section{Lithography}

For research purposes, e-beam lithography is the workhorse for the fabrication of photonic nanostructures. However, this technique is ill scalable to commercial applications. Therefore we explore the possibilities to use deep UV lithography for this purpose. Lithography at a wavelength of $248 \mathrm{~nm}$ is now the mainstream fabrication tool for high-end CMOS, and we used this for the majority of our experiments. For this, we used an ASML PAS5500/750 stepper connected to an automated track for preprocessing (coating and baking) and postprocessing (baking and developing).

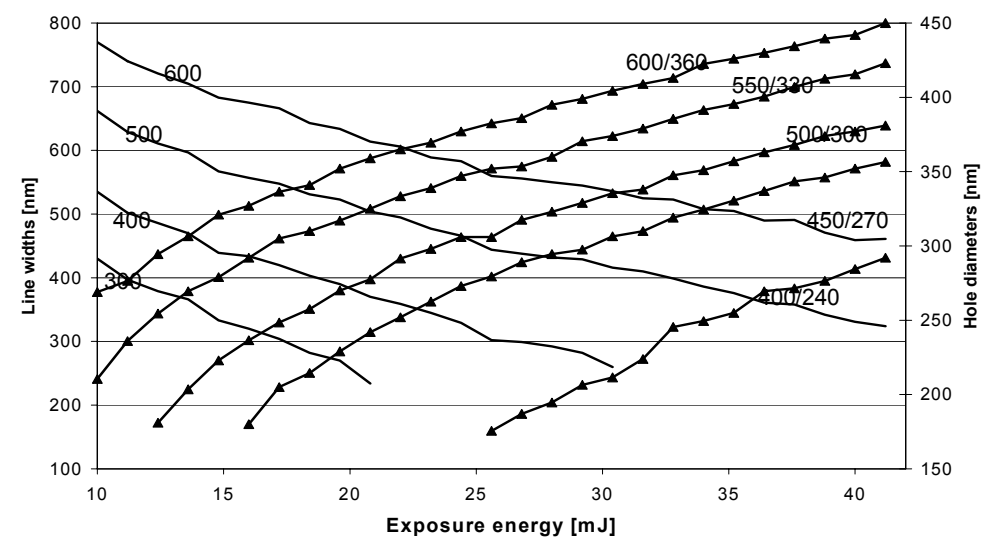

FIGURE 4. Hole sizes and line widths after lithography for various design dimensions as a function of lithography energy dose. For increasing dose, the lines narrow down and the holes grow.

Photonic nanostructures typically consist of a variety of structures. While for CMOS processes different types of structures are fabricated separately, alignment requirements for photonics make this impossible: All nanostructures should be printed in the same lithography step. However, this is not trivial, as not all types of structures print on target for the same lithography conditions. Especially the exposure dose has a significant influence on the feature size. Figure 4 shows the feature size for both isolated lines (like photonic wires) and holes in a triangular lattice (like photonic crystals) for different design values. We can see that the hole size increases with higher doses, while the line width decreases. As both structures are very different, lines being an isolated dark feature in a light field, and a photonic crystal being dense light features against a dark background, the dose-to-target will differ significantly. To fabricate both simultaneously, a bias needs to be applied to one or the other. This is the easiest for lines, being isolated features. Depending on the line width and the photonic crystal parameters, lines should be made between 10 and $100 \mathrm{~nm}$ broader than originally designed to print together with photonic crystals.

However, varying the exposure dose can also be used to lithographically change the structure parameters. As we can print many dies onto a $200 \mathrm{~mm}$ wafer with different 
exposure conditions, this gives us the ability to fabricate a number of different line widths and hole sizes in a single processing run, with a single mask. As can be seen from Fig. 4, lithography gives us a large range of feature sizes for a given design, while the variation is gradual enough to guarantee reproducible processing.

We can see that deep UV lithography is able to pattern nanophotonic structures, including photonic crystals. The process has good reproducibility, and by changing the exposure dose we can tune the photonic crystal parameters.

\section{Optical proximity effects}

While photonic wires are relatively simple structures, photonic crystals are superdense periodic structures with feature sizes close to the illumination wavelength. This causes neighboring holes to interfere with each other during lithography. Because of this, holes in a photonic crystal may interfere constructively and print larger or interfere destructively and print smaller than solitary holes. In uniform lattices, this effect is not noticeable, as the illumination energy will be chosen to print the holes in the lattice on target. However, at the boundaries of the lattice, or near defects like a waveguide or cavity, some holes lack neighbors and will therefore print differently than their counterparts in the bulk of the lattice. This phenomenon is described as optical proximity effects (OPE). An example is given in Fig. 5: although it's hard to see visually, the holes near the line defect are $40 \mathrm{~nm}$ smaller than the holes in the bulk, and in the corner this effect is even worse, with the corner hole being $70 \mathrm{~nm}$ smaller. As in photonic crystals the behavior of the component is largely determined by the shape of the defect, optical proximity effects are a very serious problem.

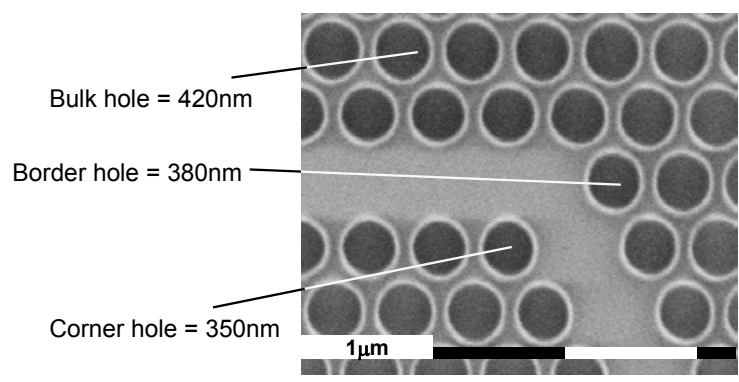

FIGURE 5. Illustration of optical proximity effects. The holes near the photonic crystal defect are printed smaller than the holes in the bulk of the lattice.

While proximity effects are also known in e-beam lithography, the serial nature of the process makes this an additive process, as the exposure dose in a certain area can only increase. For deep UV lithography, this is not the case, as the whole structure is defined in one illumination step, and the patterns can therefore interfere. This makes that OPE can be either constructive or destructive and are very hard to model accurately.

To correct for OPE, the features on the mask should be altered. This is illustrated in Fig. 6. Holes near a lattice defect are printed smaller, and are therefore enlarged on the mask. It is evident that a good understanding of the OPE is necessary to design the structures with optical proximity correction (OPC). 

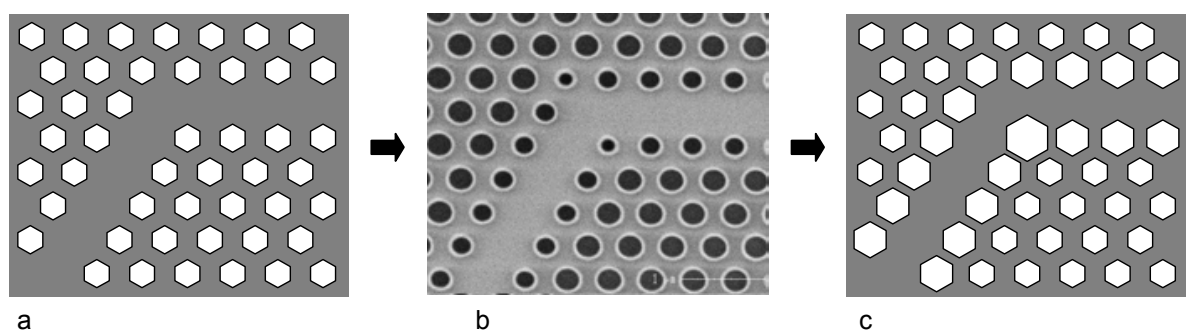

FIGURE 6. Principle of optical proximity corrections for photonic crystal structures. When uncorrected, the design on the mask (a) will print the defect holes differently than the bulk holes (b) due to optical proximity effects. To correct this, the design on the mask should be altered in advance (c).

To characterize optical proximity effects and the needed corrections in photonic crystal, we have designed a structure consisting of various photonic crystal waveguides along with a large number of bends, cavities and other possible components. We then repeated this structure on a mask with many variations of bulk hole sizes, and corrections on corners and borders. This makes it possible for us to measure the optical proximity effects and the required corrections directly and implement them on future mask designs.

\section{Etching}

For etching the Silicon-on-insulator we could also make use of CMOS processing facilities. In our case, this consisted of a multi-chamber LAM A6 platform with various process modules for the different etch processes. The wafer can be transferred between etch chambers without disrupting the vacuum. For the Silicon top layer we used an ICP low pressure/high density etch system with a chemistry based on $\mathrm{Cl}_{2} / \mathrm{HBr} / \mathrm{He} / \mathrm{O}_{2}$. For the buried oxide, a dual frequency, medium pressure/medium density etch system with a $\mathrm{CF}_{4} / \mathrm{CHF}_{3}$ chemistry was used.

Etching through the buffer layer of $1 \mu \mathrm{m}$ with only the photoresist as an etch mask proved quite a challenge. As shown in the top row of Fig. 7 and in the left part of Fig. 8 , we succeeded in etching $800 \mathrm{~nm}$ into the buried oxide, but at the cost of significant sidewall roughness.

\section{Sidewall Roughness Reduction}

Rough surfaces cause scattering. Because the amount of scattering is proportional to the square of the difference in dielectric constant on the surface interface, the effect is dramatic for high-contrast material systems, like Silicon-on insulator [1].

Sidewall roughness is caused by both lithography and etching. The exact origin is still under debate however. Basically, roughness already exists after development of the resist. As this resist is used as the etch mask for both the Silicon and silica etch, the roughness is transferred to the etched sidewalls. Sidewall roughness in the resist can be attributed to a number of causes including roughness induced by the aerial image profile [4] and by the chemical properties and reaction in the chemically amplified resists used in deep UV lithography [5]. Depending on the etch chemistry and 
conditions, this roughness can be enhanced or smoothened and new roughness can be created during etching.

To reduce these scattering losses, one can either avoid creating rough surfaces, or smoothening existing surfaces. It has already been shown that thermal oxidation of Silicon-on-insulator waveguides can smoothen the sidewalls of both photonic crystal waveguides [6] and photonic wires [7,8]. However, while oxidation can improve the roughness in the top Silicon layer, it has little or no effect on the underlying oxide layer. Therefore, light can still be scattered by the roughness in the cladding.

Instead, we chose not to etch the buried oxide. By leaving out the second etch step, the sidewall roughness was drastically reduced. This can be seen in Fig. 7 and the detail in Fig. 8. The sidewall roughness is almost completely eliminated.
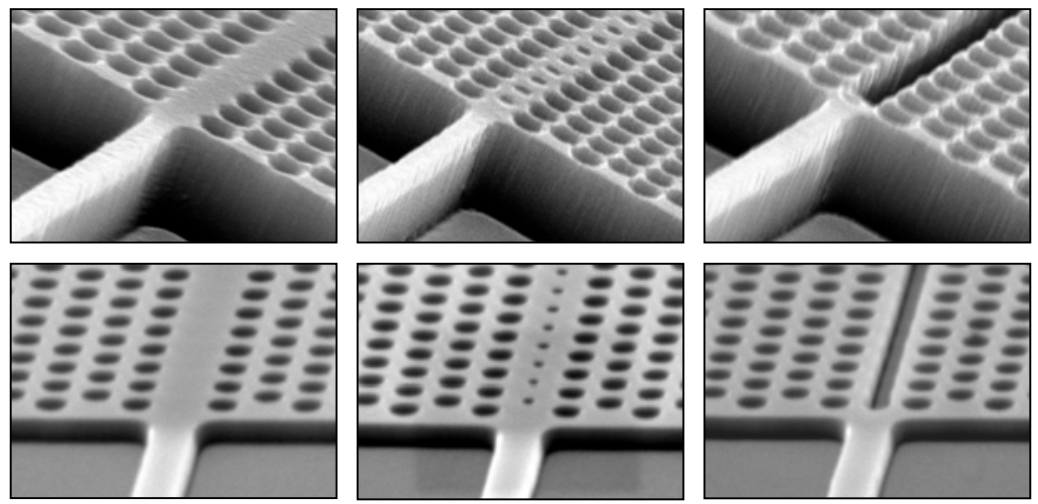

FIGURE 7. Photonic crystal waveguides fabricated with deep UV lithography and dry etching. Top row: 3 waveguide designs fabricated with deep etching, through both top Silicon layer and oxide. Bottom row: The same designs, but fabricated with only oxide etch.
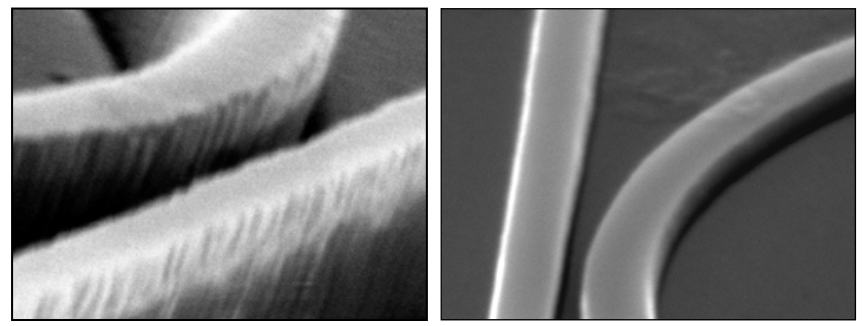

FIGURE 8. Detail of the coupling section between a straight photonic wire and a ring resonator. Left: Deep etch through both Silicon and oxide. Right: Silicon-only etch. It is clear that the deep etching causes significantly more sidewall roughness.

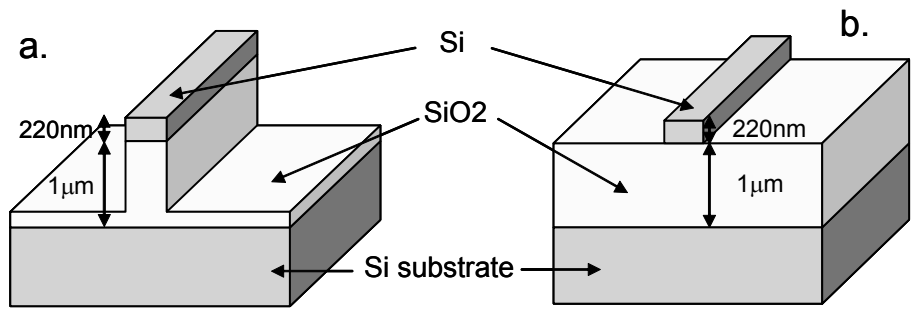

FIGURE 9. Schematic structure of a deep (a) and shallow (b) etch photonic wire. 


\section{PHOTONIC WIRES IN SILICON-ON-INSULATOR}

In this section, we will review the results of loss measurements of fabricated photonic wires. These are nanophotonic waveguides using TIR as the confinement method. We will first discuss the general properties of our waveguides, then review the causes of propagation loss and finally discuss the propagation loss measurement method and results we achieved [9].

Figure 9 shows the cross-section of a photonic wire. SEM pictures of our photonic wires fabricated using the deep UV lithography and dry etching processes described in the previous section are shown in Fig. 8. Both structures with deep etching and structures with Silicon etch only were fabricated. Simulations show that these wires are single-mode up to a width of about $610 \mathrm{~nm}$.

These wires and structures built with them will inevitably be polarization dependent, an aspect that is typical for nanophotonic waveguides and structures. The shape of the Silicon core in our structures leads to TE-like and TM-like polarized guided modes with very different propagation characteristics. The real part of the propagation constants or effective index are very different, therefore it is almost impossible to design structures that work in the same way for both polarizations. Also, TM-like polarized modes suffer much higher substrate leakage and scattering losses at sidewall roughness. The $2 \mathrm{D}$ vertical fiber couplers described in the last section are a way to eliminate this problem.

\section{Origins of propagation loss}

The propagation loss of a straight photonic wire in Silicon-on-insulator is caused by leakage to the substrate and scattering at sidewall roughness. The absorption in Silicon and silica is near zero in the wavelength region of interest.

Because the same material is used for waveguide core and substrate, no truly guided modes exist in this layer stack. The exponential tail of the modal field extends into the substrate resulting in radiation losses. The losses however decrease exponentially with increasing silica layer thickness. Therefore, one should use a cladding layer of high enough thickness. Using a mode-matching tool [10], we calculated the leakage loss of the fundamental mode in a slab waveguide as a lower boundary for the leakage losses in narrow wires [2]. Based on these calculations, SOI wafers with $1 \mu \mathrm{m}$ oxide were chosen for our experiments.

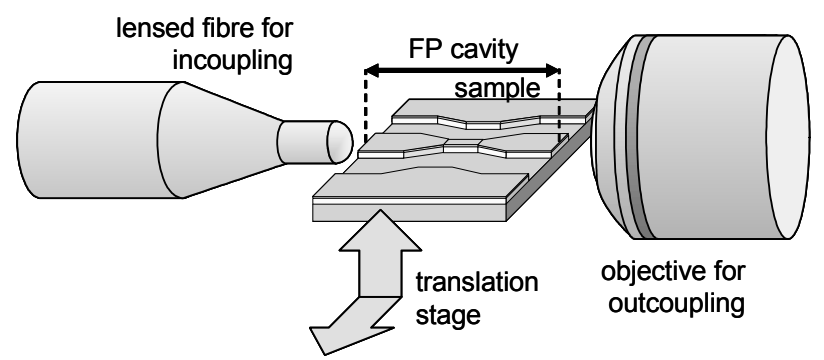

FIGURE 10. Overview of the measurement setup. Light is coupled into a $3 \mu \mathrm{m}$ broad ridge via a lensed fiber. The ridge is tapered down to the narrow wire and then again tapered up to $3 \mu \mathrm{m}$ for outcoupling to an objective. 


\section{Wire loss measurements}

A schematic overview of the measurement setup is shown in Fig. 10. We measure the transmission spectrum of the cavity formed by the two cleaved facets of a waveguide comprising $3 \mu \mathrm{m}$ wide access sections and a narrow wire in the centre. The access ridges are connected to the wire using a $300 \mu \mathrm{m}$ long taper. Light is coupled into the waveguide using a lensed fiber and coupled out to a power meter using an objective. The total loss of such a cavity is given by

$$
L_{\text {tot }}=L_{\text {waveguide }}+L_{\text {mirrors }}=10 \log \frac{\sqrt{S}-1}{\sqrt{S}+1}
$$

in which $S=T_{\max } / T_{\min }$ is the peak-to-valley ratio of the Fabry-Pérot transmission spectrum. By comparing cavities with different lengths of the narrow wire, the wire loss can be found. Figure 11 shows the loss of such a cavity, logarithmically plotted as a function of the length of the photonic wire. The wire in this example is $450 \mathrm{~nm}$ wide, no oxide etching was done. The slope of this curve gives the loss in $\mathrm{dB} / \mathrm{cm}$ of the narrow wire. The intercept of the fitted line and the vertical axis gives us the total loss of the other cavity elements (facets, broad ridges and tapers).

A $500 \mathrm{~nm}$ wide deeply etched wire has a propagation loss of $30 \mathrm{~dB} / \mathrm{mm}$, for $600 \mathrm{~nm}$ width this decreases to $6 \mathrm{~dB} / \mathrm{mm}$. The wire losses without oxide etch are much lower. Figure 12 shows the wire loss versus wire width around $1550 \mathrm{~nm}$ wavelength for shallow etching. For a wire width of $500 \mathrm{~nm}$, we measured a propagation loss of $0.24 \mathrm{~dB} / \mathrm{mm}$. For narrower wires, losses increase dramatically. SEM inspection of the structures shows that narrower wires have more roughness in absolute terms; therefore scattering losses grow faster than expected with decreasing waveguide width.

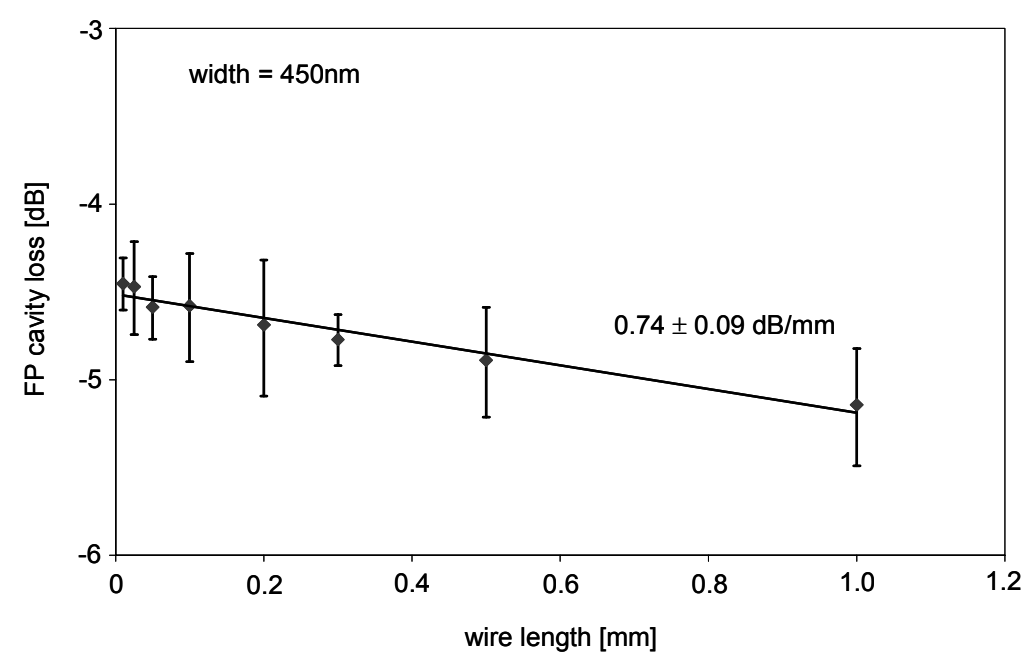

FIGURE 11. Losses of the cavity formed by the cleaved facets, narrow wire and in- and out-coupling structures, as a function of narrow wire length. The width of the wire is $450 \mathrm{~nm}$. The slope of the fitted line gives the loss of the narrow wire, the intercept with the vertical axis gives the combined losses of the in- and out-coupling structures and the facet reflectivity. 


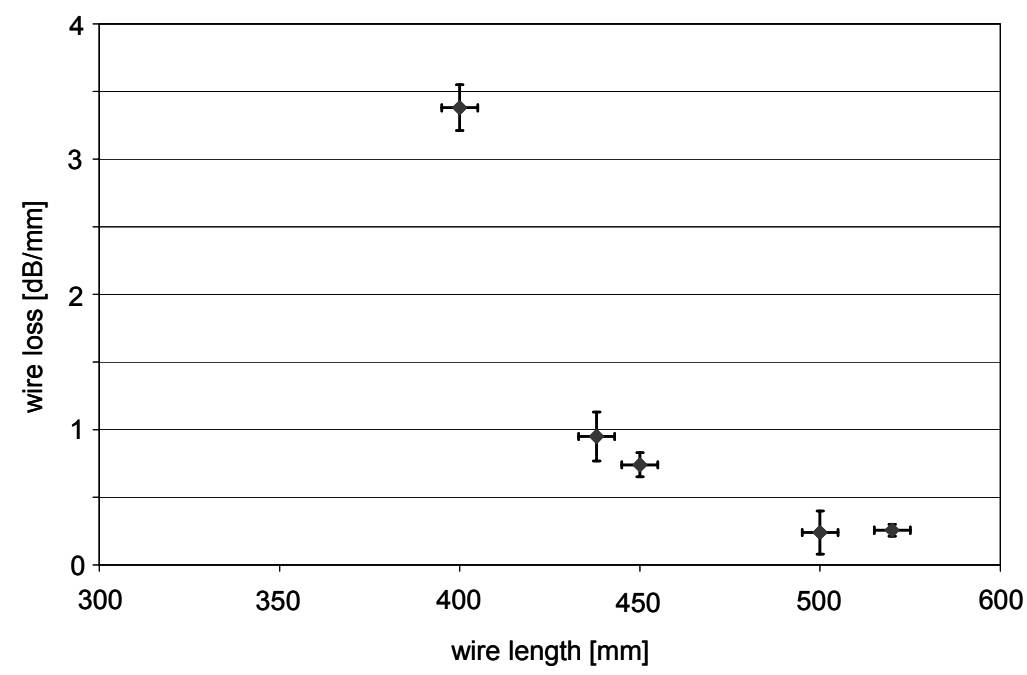

FIGURE 12. Wire loss as a function of wire width. The exponential change of losses is in agreement with sidewall roughness and substrate leakage as the loss mechanisms.

\section{RING RESONATORS}

Ring resonators can provide building blocks for a large number of functional components on a photonic IC, including various types of filters. For WDM purposes for instance, ring resonators with ultra-small radius could provide for add-drop filters operating over a large wavelength range, as they have a large free spectral range (FSR). Waveguides with a high lateral index contrast can lead to very compact bends with negligible fundamental bending losses, making such small resonators possible. The number of wavelength channels that can be handled at given intra-channel (adddrop) and inter channel crosstalk requirements is determined by the finesse of the resonator, which is limited by the cavity loss. We fabricated ring and "racetrack" resonators, symmetrically coupled to straight input and output waveguides. Figure 13 shows a SEM picture of a fabricated ring resonator. In this section, we will review the properties of these resonators and discuss the measurement results.

Coupling between the ring resonator and waveguides is achieved using a directional coupler-type structure. The coupling gap is an important parameter as it influences finesse, insertion loss and crosstalk to a great extent. The finesse decreases with increasing coupling ratio. Only at a critical coupling ratio that compensates exactly for all cavity loss, all power is extracted from the input waveguide at resonance [11] and zero add-drop crosstalk can be achieved. As our waveguides have very high confinement, the gap needs to be very small to achieve a high enough coupling to operate in the optimal regime. With our DUV lithography fabrication technique, gaps down to $200 \mathrm{~nm}$ width can be made with good reproducibility. To enhance the coupling, a racetrack resonator can be used, as shown in Fig. 13. One effect that should be taken into account (both for rings and racetracks) is the narrowing of the waveguides in the coupling region due to optical proximity effects [2]. 
As pointed out in the first section, for high index contrast structures, the resonance wavelength of tiny resonators is highly dependent on the exact optical path length in the ring at resonance wavelength. A $1 \mathrm{~nm}$ change in optical path length will result in a shift in resonance wavelength of the same order, which is unacceptable for dense WDM applications. Therefore, one needs tight control over the waveguide effective and group indices.

The resonator finesse is limited by cavity losses as mentioned above. By characterization of fabricated rings and fitting the results to calculated characteristics, we can get insight into the relative contribution of the several loss mechanisms. Theoretically, intrinsic bending loss and scattering at sidewall roughness contribute to the total loss. Simulations with a 3D mode-matching tool [12] show that the intrinsic bending loss is mostly due to higher substrate leakage. As long as the radius is not too small, intrinsic bend losses - that would in theory also occur without substrate - are negligible. Also, our calculations show that the loss due to the modal mismatch between straight and bent waveguides in a racetrack resonator and the tapering loss due to the narrowing of the wires in the coupling region are very small.
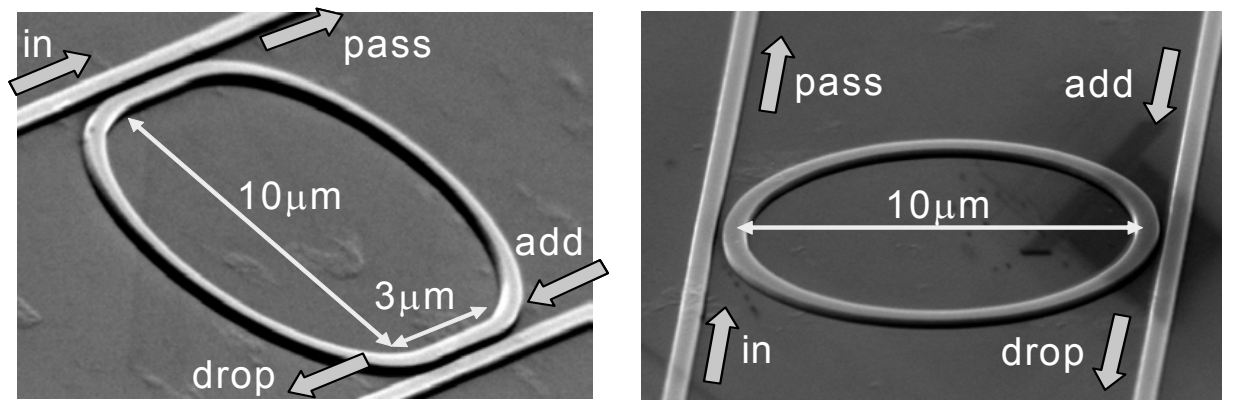

FIGURE 13. SEM picture of a racetrack (left) and ring (right) resonator with Silicon etch only. At the resonance wavelengths, power is transferred from the input port to the drop port and from the add port to the pass port.

\section{Measurement results}

Following AR-coating, we measured the transmission spectrum from the "pass" and "drop" ports as shown in Fig. 13, using the measurement method described in the previous section. We measured a ring resonator with $5 \mu \mathrm{m}$ radius. This ring has a FSR of $17 \mathrm{~nm}$ and a FWHM of $0.19 \mathrm{~nm}$, so a finesse of 89 (Q-factor of over 8000 ) is achieved. The add-drop cross-talk is very high however, due to the low coupling ratio, making the ring useless as an add-drop filter. Figure 14 shows the pass and drop port transmission for a racetrack resonator with $5 \mu \mathrm{m}$ radius and coupling section of $3 \mu \mathrm{m}$ length. Around $1550 \mathrm{~nm}$, the resonator has a FWHM of $0.48 \mathrm{~nm}$, a finesse of 28 (Q>3200) and an add-drop crosstalk of around 20dB.

By fitting the theoretical model of the resonator to the experimental data, we extracted the group index of the cavity waveguide, the coupling factor and the propagation loss in the cavity. 
A fit of the free spectral range (FSR) over a large enough number of periods in the spectrum, gives us the group index of the cavity waveguide. This is an averaged value over the ring however: the waveguides become smaller in the coupling region due to optical proximity effects of the DUV lithography and the straight and curved waveguides in a racetrack resonator have a different group index.

For the spectrum in Fig. 14, the FSR of $13.7 \mathrm{~nm}$ leads to a group index of 4.8 , showing the large waveguide dispersion as the effective index is around 2.8 . Measurements of racetrack and ring resonators with a range of waveguide and gap widths show that the effective index of the guided mode in the ring is extremely dependent on waveguide width. We measured a group index change of about 1 to 2 per cent with a waveguide width change of 1 to 2 per cent $(5 \mathrm{~nm})$. This leads to resonance wavelength shifts of $15 \mathrm{~nm}$, confirming the fact that the tolerable error on the optical path length is of the same order as the tolerable resonance wavelength error (see first section).

Around $1550 \mathrm{~nm}$, the amplitude coupling factor of the coupler is 0.3 , which is fairly optimal. Higher coupling at longer wavelengths leads to a decreasing finesse, lower coupling leads to higher add-drop crosstalk.

The extracted cavity roundtrip propagation loss for the resonator from Fig. 14 is about $0.2 \mathrm{~dB}$, or $5 \mathrm{~dB} / \mathrm{mm}$. The loss of an equivalent combination of straight waveguides is about $1 \mathrm{~dB} / \mathrm{mm}$. This extra loss is due to higher substrate leakage due to the bending as mentioned above, and to a minor extent from mode mismatch between the straight and bent racetrack sections and from higher roughness in the coupling regions due to a worse aerial image profile.

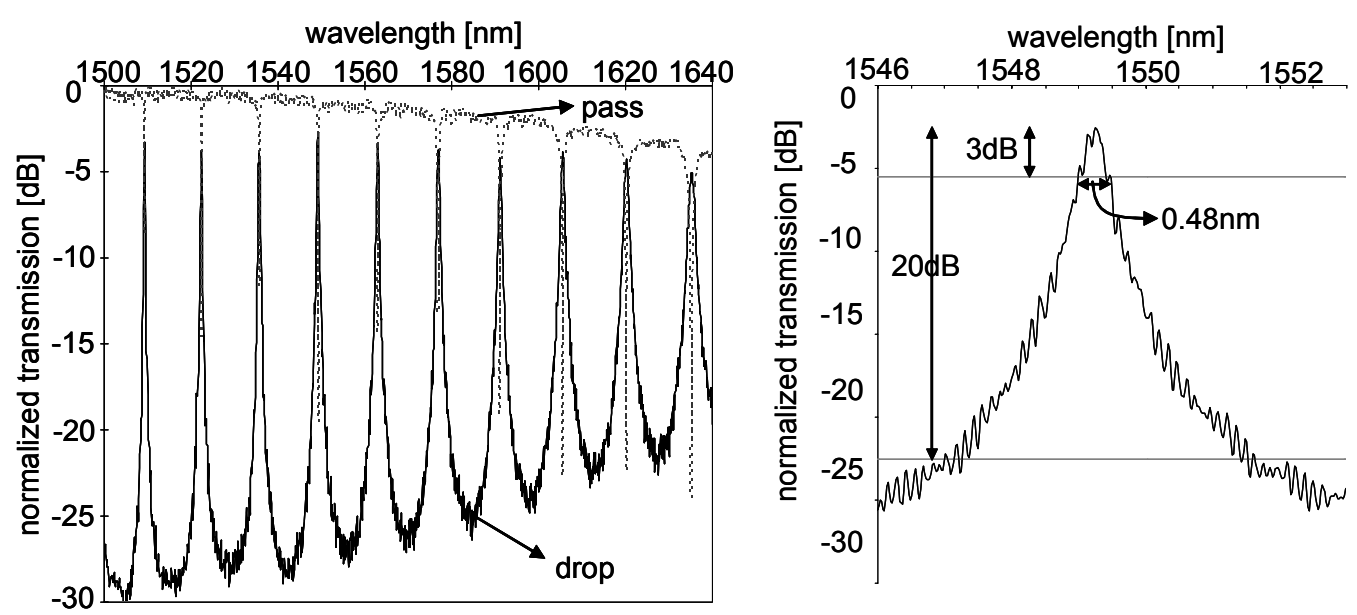

FIGURE 14. (left) drop and pass port spectrum of a racetrack resonator with $5 \mu \mathrm{m}$ radius and $3 \mu \mathrm{m}$ long coupling section, measured with AR coating on the samples facets. (right) detail of the resonance around $1550 \mathrm{~nm}$. The FSR is $23.7 \mathrm{~nm}, \mathrm{FWHM}$ is $0.48 \mathrm{~nm}$, leading to a Q factor higher than 3200 (finesse 28 ). Both pass and drop spectra are normalized on the maximum pass transfer. The coupling and cavity loss becomes higher at higher wavelengths, leading to lower Q factors. 


\section{THE INTERFACE TO THE OUTSIDE WORLD: EFFICIENT COUPLING TO FIBER}

The interface between nanophotonic waveguides and optical fibers is an important problem. Single-mode optical fibers, used in optical communications, typically have a core diameter and mode size of about $10 \mu \mathrm{m}$. The huge mismatch between the mode size of an optical fiber and a nanophotonic waveguide complicates the coupling of light from one to the other. Simple butt-coupling would result in more than $20 \mathrm{~dB}$ coupling loss.

We will discuss several published methods for achieving efficient coupling to fiber. All these methods use some kind of integrated spot-size converter that is made on the same chip as the nanophotonic waveguides. There exist also non-integrated solutions for improving the coupling efficiency, but at the cost of very difficult alignment requirements. In the next paragraphs we will discuss tapered spot-size converters and grating based couplers. Last but not least, we will discuss how to deal with the polarization dependence of nanophotonics.

\section{Tapered spot-size converters}

An adiabatic taper can convert the small mode from the nanophotonic waveguide into a larger mode that couples better to fiber. A taper is typically a waveguide of which the dimensions change along the propagation direction, creating a transition from a small to a large mode size. If the variation is slow enough, the fundamental mode of the input waveguide is converted into the fundamental mode of the output waveguide with $100 \%$ efficiency and the taper is called adiabatic.

The theoretically most straightforward solution to our problem is a 3-D taper, this is a structure that is tapered both laterally and vertically (Fig. 15). Because of the huge difference in mode size between a nanophotonic waveguide and a fiber, such a taper is very long, almost $1 \mathrm{~mm}$. Also the fabrication process of this 3-D structure is rather complicated and published experimental results achieve only $-4.5 \mathrm{~dB}$ conversion efficiency [13].
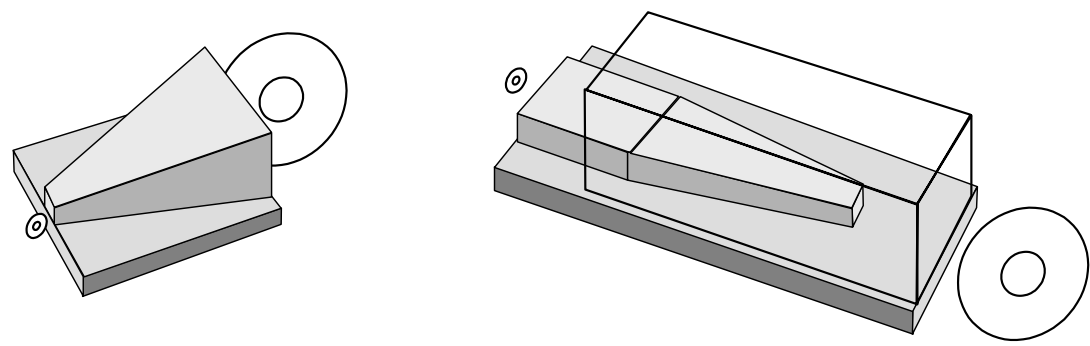

FIGURE 15. Examples of tapered spot-size converters : A 3-D taper (left) and a 2-D tapered spotsize converter (right). Please note that the taper angles are strongly exaggerated, the length of the taper is typically a few $100 \mu \mathrm{m}$ up to $1 \mathrm{~mm}$ 
A more elegant solution is the use of a 2-D taper (tapered only laterally) to achieve 3-D spot-size conversion. An example structure is shown in Fig. 15. The nanophotonic waveguide is tapered; its width is reduced to a few tens of nanometers. This forces the mode out of the Silicon nanophotonic waveguide into another fiber adapted waveguide that is either on top or below the Silicon. This fiber adapted waveguide can be a thick Silicon dioxide layer or a polymer waveguide that is made on top of the Silicon. These structures can be fabricated with conventional processing techniques, although very high resolution lithography is required for the taper tip.

A coupling loss of $0.8 \mathrm{~dB}$ between a $300 \mathrm{~nm} \times 300 \mathrm{~nm}$ Silicon waveguide and a standard single-mode fiber has been demonstrated by NTT [14]. To our knowledge this is the highest coupling efficiency experimentally demonstrated. They use a polymer fiber adapted waveguide and a $200 \mu \mathrm{m}$ long taper with a tip width of $60 \mathrm{~nm}$. The polymer waveguide is connected to $4 \mu \mathrm{m}$ mode size high index fiber. This fiber is connected to normal fiber with very low loss, but the use of fiber with a smaller mode size increases the alignment sensitivity.

\section{Grating Couplers}

A completely different approach is the use of a grating to couple top-illuminating light into the submicrometer thin waveguides. Because light is coupled in and out from the top, there is no need to cleave or polish devices and it is possible to test the circuits 'wafer-scale', before packaging. Grating couplers in SOI have been demonstrated some time ago [15], these couplers have a high efficiency but at the cost of a very narrow bandwidth. We have demonstrated that a very compact grating based coupler can also be used to couple light to nanophotonic waveguides with a bandwidth of several tens of nanometers [16]. The grating takes care of the vertical spot-size conversion. For the horizontal spot-size conversion, a traditional adiabatic taper is used to connect a $220 \mathrm{~nm} \times 12 \mu \mathrm{m}$ waveguide to a $220 \mathrm{~nm}$ x $500 \mathrm{~nm}$ waveguide.
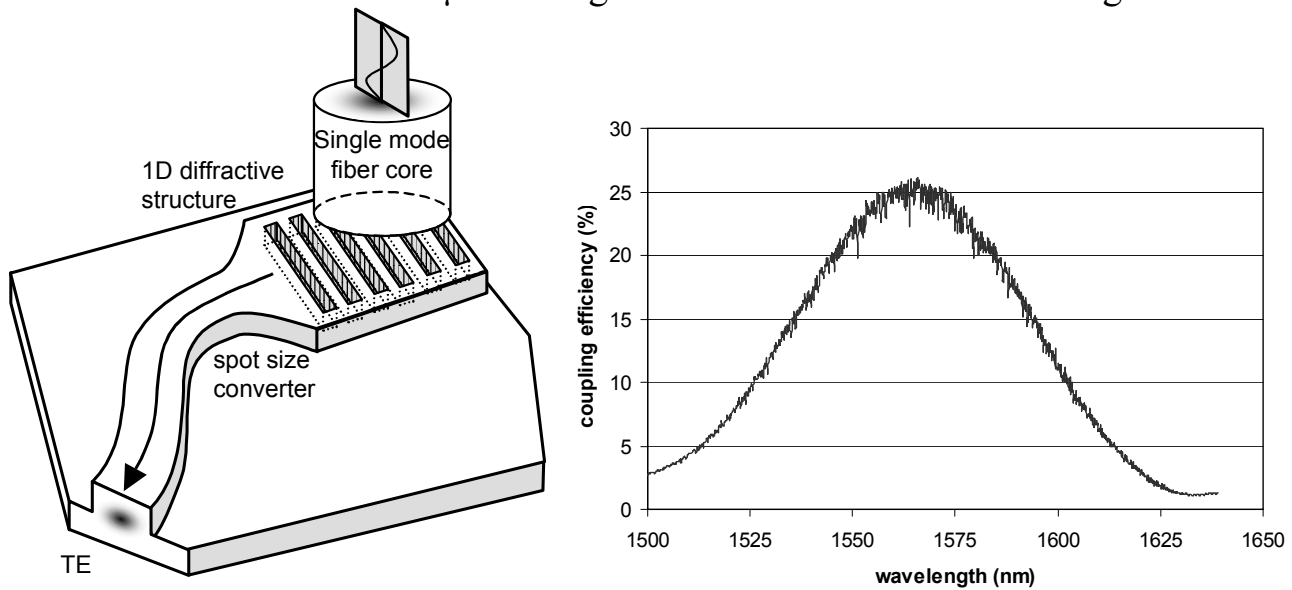

FIGURE 16. Grating coupler principle (only the core of the fiber is shown) and measured coupling efficiency to single-mode fiber. 
We have experimentally demonstrated $25 \%$ coupling efficiency from a single mode-fiber to a $220 \mathrm{~nm}$ thin Silicon waveguide for TE-polarized light (Fig. 16). The $1 \mathrm{~dB}$ bandwidth is $35 \mathrm{~nm}$ and the $3 \mathrm{~dB}$ bandwidth $65 \mathrm{~nm}$. The grating has a period of $620 \mathrm{~nm}, 50 \%$ fill factor and is etched $90 \mathrm{~nm}$ into the top Silicon layer. A fiber alignment error of $\pm 1 \mu \mathrm{m}$ in any direction results in less than $0.5 \mathrm{~dB}$ additional loss.

The efficiency of this coupler can be increased by using SOI material with a buried reflector. It is possible to fabricate SOI with a 2-pair DBR mirror below the waveguide [17], and thanks to the high index contrast, a 2-pair mirror is sufficient. Unfortunately this material is not commercially available. Although the achieved efficiency isn't yet sufficient for commercial applications, the couplers are already useful for wafer-scale testing of devices.

\section{Polarization}

Most nanophotonic waveguides and components are polarization sensitive. It is very difficult, if not impossible, to make a polarization independent photonic integrated circuit with these structures. Because the polarization of light coming from an optical fiber is random, polarization independent operation is required in optical communication systems. One solution is to use a polarization splitter that splits the light from the fiber in two orthogonal polarization states, followed by a polarization converter that converts one of these states into the other. Two identical circuits are then used on chip. At the other end, another polarization converter and splitter are used to combine both circuits back into a fiber. However it is very difficult to integrate the polarization splitter/converter on the same chip as the nanophotonic circuits and if the splitter/converter is not integrated, the advantage of large-scale integration of nanophotonics is lost. In the next paragraph we will describe a different polarization diversity approach that can be completely integrated with nanophotonic integrated circuits in SOI. It is based on a grating coupler that is used as a polarization splitter [18].
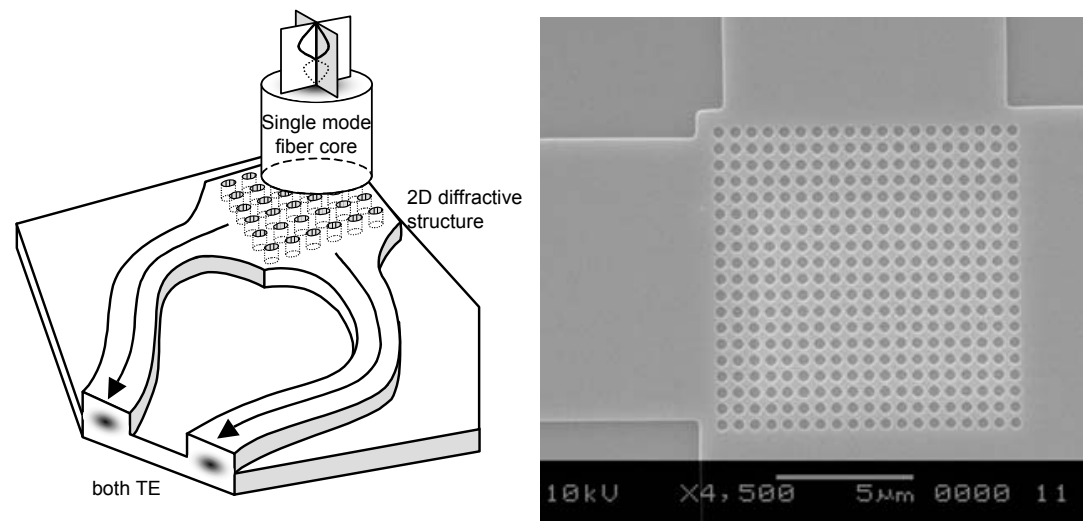

FIGURE 17. 2-D grating coupler used as a polarization splitter, SEM top view (right)

The coupler uses a 2-D grating and two waveguides (Fig. 17). The grating is a square array of holes and the angle between the two waveguides is 90 degrees. 
Because of the polarization sensitivity of the grating coupler, only the fundamental TE-mode of both waveguides can couple efficiently to fiber (the efficiency for the fundamental TM waveguide mode is much lower). When using this device as input coupler, the light from the fiber couples to both waveguides. The coupling ratio depends on the state of polarization, but the total coupling to both waveguides together does not. We have demonstrated an extinction ratio $>18 \mathrm{~dB}$ in the wavelength range $1530-1560 \mathrm{~nm}$. The coupling efficiency was $20 \%$ [18].

The two waveguides from the coupler can be connected to two identical circuits that function only for TE-polarization. When using the grating coupler as input and output coupler, the transmission from input to output fiber is independent of the state of polarization in the fiber. The coupler performs the function of polarization splitter/converter as described in the previous paragraphs and can be easily integrated.

\section{CONCLUSION}

Nanophotonic components, wavelength-scale structures using high index contrast, are the key to further integration of photonic IC's. We have discussed the major argument to this: these waveguides and cavities support only few resonant electromagnetic modes. An important implication of using high index contrast structures we have illustrated is that nanometer wavelength precision of wavelengthselective components can only be achieved with nanometer geometry precision.

We have shown that deep UV lithography with dry etching is a very good candidate for commercial fabrication of nanophotonic structures. However, a detailed process characterization and optimization is needed to reliably produce high-quality components. For lithography, the necessary biases and optical proximity corrections are needed before mask fabrication, and for deep etching one has to care about the sidewall roughness. For optical proximity effects, we have fabricated test structures to measure the necessary corrections. In order to keep the sidewall roughness to a minimum, we chose to etch only the top Silicon layer and not etch the buried oxide.

We have fabricated single-mode photonic wires, waveguides confining light by total internal reflection, in Silicon-on-Insulator with propagation losses as low as $0.24 \mathrm{~dB} / \mathrm{mm}$. Therefore this technology allows for low-loss interconnects and building functional components on dense integrated photonic IC's. We demonstrated this through the fabrication of ring resonators with low crosstalk figures and high finesse.

To tackle the problem of interfacing nanophotonic circuits with fiber, we demonstrated vertical fiber couplers with $25 \%$ efficiency and $65 \mathrm{~nm}$ bandwidth. A $2 \mathrm{D}$ fiber coupler integrates both polarization splitting and polarization conversion functionality in one compact device, solving the inevitable polarization dependency problem in high index contrast nanophotonic circuits. 


\section{ACKNOWLEDGMENTS}

Part of this work was done in the context of the IST-PICCO project supported by the European Union.

Part of this work was carried out in the context of the Belgian IAP PHOTON network.

W. Bogaerts and P. Dumon thank the Flemish Institute for the industrial advancement of scientific and technological Research (IWT) for a specialization grant.

J. Van Campenhout was supported by the Flemish Fund for Scientific Research (FWO-Vlaanderen) through a doctoral fellowship.

D. Van Thourhout acknowledges the Belgian Federal Office for Scientific, Technical and Cultural Affairs for financial support.

P. Bienstman acknowledges the Flemish Fund for Scientific Research (FWOVlaanderen) for a postdoctoral fellowship.

The authors would also like to thank D. Vangoidsenhoven for the wafer exposures and R. de Ruyter and J. Mees for their work on the mask design.

\section{REFERENCES}

1. Bogaerts W., Bienstman P. and Baets R., Opt. Lett. 28(9), p. 689, 2003

2. Bogaerts W., Wiaux V., Taillaert D., Beckx S., Luyssaert B., Bienstman P. and Baets R., IEEE J. Sel. Top. Quantum Electronics, 8(4), p. 928, 2002

3. SOITEC's Unibond(R) process, Microelectronics Journal, vol. 27 (4-5) p. R36, 1996

4. Williamson M. and Neureuther A., "Enhanced, quantitative analysis of resist image contrast upon line edge roughness", Proceedings of the SPIE Vol. 5039, June 2003

5. Fukuda H., Jpn. J. Appl. Phys, Vol. 42 (2003) No. 6B pp. 3748-3754, June 2003

6. J. Arentoft, T. Sondergaard, M. Kristensen, A. Boltasseva, M Thorhauge and L. Frandsen, Electron. Lett. $38(6)$, p. 274-275, 2002

7. K. K. Lee, D.R. Lim, L.C. Kimerling, J. Shin, F. Cerrina, Opt. Lett. 26(23), p.1888, 2001

8. T. Tsuchizawa, T. Watanabe, E. Tamechika, T. Shoji, K. Yamada, J. Takahashi, S. Uchiyama, S. Itabashi and H. Morita, "Fabrication and evaluation of submicron-square Si wire waveguides with spot-size converters", LEOS Ann. Meeting 2002, Glasgow, UK, p.287

9. Dumon P., Bogaerts W., Van Campenhout J., Wiaux V., Wouters J., Beckx S. and Baets R., "Low-loss singlemode photonic wires and ring resonators in Silicon-on-Insulator", LEOS Ann. meeting, Tuscon, AZ, 26-30 Oct. 2003.

10. Bienstman P. and Baets R., Opt. Quant. El., Vol. 33, p. 327, Jan. 2001

11. Yariv A., IEEE Phot. Tech. Lett., 14(4), p. 483, April 2002

12. Fimmwave/FimmProp3D, see www.photond.com

13. Frisch M.B., Fijol J.A., Fike E.E., Jacobson S.A., Keating P.B., Kessler W.J., LeBlanc J., Bozler C., Fritze M., Keast C., Knecht J., Williamson R. and Monalatou C., "Coupling of single mode fibers to planar Si waveguides using vertically tapered mode converters", paper IFB2-1 in proceedings of the Integrated Photonics Research conference, Vancouver, Canada, July 14-19, 2002.

14. Shoji T., Tsuchizawa T., Watanabe T., Yamada K., and Morita H., Electronics Letters 38, 1669-1670 (2002).

15. Emmons R.M. and Hall D.G., IEEE J. Quantum Electron. 28, 164-175 (1992).

16. Taillaert D., Bogaerts W., Bienstman P., Krauss T.F., Van Daele P., Moerman I., Verstuyft S., De Mesel K. and Baets R., IEEE J. Quantum Electron. 38, 949-955 (2002).

17. Emsley M.K., Dosunmu O., Unlu M.S., IEEE J. Sel. Top. Quantum Electron. 8, 948-955 (2002).

18. Taillaert D., Chong H., Borel P., Frandsen L., De La Rue R. and Baets R., IEEE Photonics Technology Letters $15,1249-1251$ (2003). 